Projets

de paysage

\section{Projets de paysage}

Revue scientifique sur la conception et l'aménagement de l'espace

18 | 2018

Paysage et didactique

\title{
Le grand paysage, révélateur des enjeux de la formation en architecture de paysage
}

L'exemple du Québec

The larger landscape, revealing the challenges of landscape architecture training - The example of Quebec

\section{Gérald Domon}

\section{OpenEdition}

Journals

Édition électronique

URL : http://journals.openedition.org/paysage/1048

DOI : $10.4000 /$ paysage. 1048

ISSN : 1969-6124

\section{Éditeur :}

École nationale supérieure du paysage de Versailles-Marseille, Institut national des sciences appliquées Centre Val de Loire - École de la nature et du paysage, École nationale supérieure d'architecture et de paysage de Bordeaux, École nationale supérieure d'architecture et de paysage de Lille, Agrocampus Angers

\section{Référence électronique}

Gérald Domon, « Le grand paysage, révélateur des enjeux de la formation en architecture de paysage », Projets de paysage [En ligne], 18 | 2018, mis en ligne le 07 juillet 2018, consulté le 28 novembre 2019. URL : http://journals.openedition.org/paysage/1048 ; DOI : 10.4000/paysage.1048

Ce document a été généré automatiquement le 28 novembre 2019.

Projets de paysage 


\section{Le grand paysage, révélateur des enjeux de la formation en architecture de paysage}

L'exemple du Québec

The larger landscape, revealing the challenges of landscape architecture training - The example of Quebec

\section{Gérald Domon}

Une comparaison, même sommaire, avec la France ou d'autres pays européens, révèle certaines singularités du Québec en matière de formation en paysage. D'abord, alors que dans différentes universités françaises, les départements de géographie offrent des formations spécialisées en paysage, au Québec, en dépit d'importantes contributions passées (Bureau, 1977 ; Raveneau, 1977), les géographes ont depuis longtemps tourné le dos au paysage. Aussi, l'offre de cours est-elle aujourd'hui marginale dans les départements de géographie. Ensuite si, en France, différentes écoles proposent des formations de paysagistes, l'École d'urbanisme et d'architecture de paysage de l'université de Montréal assume seule cette formation pour l'ensemble du Québec. L'accueil fréquent d'étudiants français (les écoles de Blois et de Bordeaux principalement) témoigne d'une équivalence certaine des enseignements offerts dans les écoles françaises et l'école québécoise: l'atelier pédagogique au cœur de la formation, insistance sur la connaissance et l'utilisation des végétaux, maîtrise des détails techniques, etc. Cela dit, les programmes (préprofessionnel de premier cycle; professionnel de second cycle) de l'université de Montréal ont, pour autre singularité, d'avoir comme finalité la formation d'architectes paysagistes. Peu usité en France et renvoyant largement à une conception et à une tradition nord-américaines, voire anglo-saxonnes, le terme architecture de paysage désigne cette discipline qui «comprend le design, la planification, la gestion et la conservation des espaces extérieurs» (Williams, 2014, p. 3). C'est dire que les programmes en architecture de paysage visent non seulement la formation de paysagistes concepteurs mais aussi de professionnels aptes à agir en tant que planificateurs et gestionnaires de paysages. 
Enfin, autre particularité, le programme professionnel est soumis à l'accréditation d'une association, l'Association des architectes paysagistes du Canada (AAPC). Cette accréditation a pour effet de doter le nouveau diplômé d'un statut qui lui permet d'être reconnu, en tant qu'architecte paysagiste, dans les autres provinces canadiennes et aux États-Unis.

2 En milieu universitaire, il s'agit là d'une situation qui, sans être unique, demeure singulière. Contrairement aux formations en géographie, en sociologie, en biologie ou en de nombreuses autres disciplines, l'identification des connaissances et des compétences à acquérir découle non seulement de la vision des responsables du programme mais aussi de celle de l'association professionnelle. De fait, c'est elle qui, à travers le processus d'accréditation, décidera ultimement du contenu du programme.

Or, qu'en est-il de ces visions? Dans quelle mesure coïncident-elles pleinement? En prenant appui, d'une part, sur une lecture de l'origine et du contenu des programmes proposés et, d'autre part, sur le récent rapport d'accréditation du programme de second cycle qui explicite la vison de la formation qu'a l'AAPC, le présent texte propose de se pencher sur ces questions. De manière plus précise, il les aborde à partir de l'exemple du grand paysage, conçu ici comme "[...] un saut d'échelle au-delà des pratiques de conception tournées vers des objets spatiaux a priori cernables tels que des jardins, des places, des parvis ou les espaces publics de quartiers » (Pernet, 2014, p. 18). Si l'intérêt de cette échelle tient notamment au fait qu'elle témoigne d'une volonté d'intervenir sur l'ensemble du territoire (Pernet, 2014) et mobilise ainsi une diversité de pratiques (création, protection, gestion, etc.), différents facteurs font en sorte que la formation en grand paysage mérite une attention particulière au Canada. Ainsi, les travaux menés à cette échelle par Ian McHarg (1969) demeurent parmi les plus significatifs réalisés par des architectes paysagistes de l'Amérique du Nord (Weller, 2017). De même, comme il ressort de l'ouvrage de synthèse L'Architecture de paysage au Canada (Williams, 2014), la prise en compte de cette échelle y est incontournable en raison de l'histoire et de la géographie du pays. Enfin, c'est à cette échelle que se traiteraient le mieux les préoccupations sociales et environnementales qui y sont les plus pressantes: changements climatiques, urbanisation débridée, réconciliation avec les autochtones, etc. (Trottier, 2017).

Les pages qui suivent expliquent, dans un premier temps, l'origine de l'atelier pédagogique en grand paysage, les principes qui le gouvernent et les limites qui ont contribué à la mise en place d'un programme de second cycle. Dans un deuxième temps, elles présentent ce programme puis le mettent en relation avec le contenu du rapport d'accréditation de l'AAPC, rapport qui met au jour la vision de la formation soutenue par cette association. Cette mise en relation soulève, dans un troisième temps, des enjeux qui, par-delà le seul exemple du grand paysage, sont au cœur de la formation et de la discipline.

\section{L'atelier pédagogique « Espace régional » comme réponse initiale à la nouvelle demande en grand paysage}

5 Depuis la mise en place du programme de premier cycle en architecture de paysage en 1968, un atelier pédagogique a, sous diverses appellations, assuré la présence du grand 
paysage dans l'enseignement. Répondant aux besoins découlant d'une forte croissance démographique et d'une société qui, à la sortie de la révolution tranquille ${ }^{1}$, se trouve en pleine modernisation, cet atelier portera prioritairement, pendant quelque 25 ans, sur l'élaboration de plans d'aménagement de sites spécifiques : parcs nationaux, ensembles récréotouristiques, infrastructures autoroutières, etc. Il ne s'agissait donc pas tant d'intervenir à l'échelle du grand paysage que sur des sites spécifiques à l'intérieur de celui-ci.

6 Un tournant significatif allait s'amorcer dans la première moitié des années 1990 alors que le maire de Saint-Étienne-de-Bolton, municipalité déjà engagée dans le Réseau québécois de villes et villages en santé, contacte l'École d'architecture de paysage ${ }^{2}$ pour amorcer une réflexion sur l'avenir de son territoire. Petit village de 377 âmes, SaintÉtienne est alors aux prises avec des difficultés multiples (déprise marquée de l'agriculture, fermeture de l'école, tensions entre villégiateurs et résidents permanents, etc.), et ce en dépit d'un cadre naturel remarquable. S'engage alors, pour la première fois au Québec, une démarche de projet pilotée par le maire et une étudiante à la maitrise (Lacasse, 1995), démarche à laquelle participeront deux cohortes successives d'étudiants de l'atelier pédagogique « Espace régional ».

7 Il faudra toutefois attendre près de dix ans, soit la mise en place de collaborations semblables avec la ville de Sutton (en 2002) puis celle de Sainte-Catherine-de-Hatley (en 2003), pour que l'Atelier acquière graduellement sa forme actuelle. De celle-ci, nous soulignerons cinq principes qui servent en quelque sorte de fondement.

- Assurer une connaissance plus juste de la ruralité et de sa diversité. Pour la majorité des étudiants, originaires pour la plupart de la ville ou de la proche banlieue, le rural demeure largement méconnu. Il est le plus souvent synonyme de paysages bucoliques figés dans le temps, de paysages de peu d'intérêt. À travers un stage sur le terrain qui précède l'atelier, il s'agit de permettre à l'étudiant de prendre une mesure plus juste du rural, de sa diversité et, du coup, de son intérêt.

- Miser sur une collaboration étroite entre université et collectivité pour offrir un cadre privilégié de formation. S'agissant d'une formation professionnelle, il importe d'enrichir la formation académique de l'étudiant en le plaçant dans un contexte s'approchant de celui de la pratique. Il faut ainsi lui permettre de saisir la complexité du milieu d'intervention et de confronter ses visions et ses propositions aux attentes des collectivités.

- Assurer un retour aux collectivités à travers des livrables appropriables. Puisque dans une telle collaboration, la collectivité apporte beaucoup à l'étudiant, il importe de lui assurer un certain retour sous des formes appropriables: expositions de travaux, construction de boîtes à outils, assistance dans la réalisation d'interventions, etc.

- Faire de l'atelier un lieu d'exploration et d'échanges réciproques entre recherche, enseignement et pratique. Une des plus grandes responsabilités d'une formation professionnelle demeure d'en arriver à former des individus qui pratiqueront encore dans 10, 20, 30 ans. Aussi gagne-t-on, pour y parvenir, à faire de l'atelier un lieu d'exploration ouvert à de nouveaux enjeux, à de nouvelles pratiques, un des moyens à privilégier étant de coupler territoire de recherche et territoire d'enseignement (Domon et al., 2006).

- Ne jamais faire deux fois la même chose. À travers le renouvellement constant des problématiques et des territoires traités, il s'agit à la fois de maintenir le caractère exploratoire de l'atelier et de permettre aux étudiants d'apporter chaque année une contribution nouvelle aux collectivités. 

déclinés de manière différente à chaque édition. Cela dit, le déroulement type est présenté dans le tableau 1.

9 En raison de la difficulté rencontrée par plusieurs individus (professionnels, étudiants, etc.) d'en arriver à comprendre ce que pouvait être le travail d'un architecte-paysagiste à l'échelle des grands paysages, l'intérêt de l'atelier a souvent été contesté lors de sa mise en place. Or, l'expérience des quinze dernières années, couplée à la multiplication des propositions de collaboration émanant des collectivités locales, a largement confirmé sa pertinence. Ainsi, en misant sur la collaboration étroite avec les collectivités locales à travers une série de va-et-vient entre ses membres (étudiants et responsables) et les représentants du milieu (élus, professionnels, citoyens), il permet, dans une certaine mesure du moins, un enrichissement mutuel à un double niveau.

niveau de la formation d'abord, les étudiants sont confrontés aux réalités politiques et économiques des collectivités tout en étant amenés, à travers la présentation publique de leurs propositions, à développer des habiletés d'un point de vue communicationnel et interactionnel, habiletés souvent négligées dans la formation (Milburn et Brown, 2003; Palea et al., 2012). Par voie de retour, les collectivités sont quant à elles exposées à une panoplie de méthodes de caractérisation des paysages et bénéficient d'un regard externe sur leur territoire. Davantage encore, les propositions des étudiants les amènent «à se libérer des contraintes du quotidien, pour rêver à ce que pourrait être le futur » (Couillard, 2016).

Tableau 1. Déroulement type d'une édition de l'atelier « Espace régional »

\begin{tabular}{|c|c|c|}
\hline & ACTIVITÉ & DESCRIPTION \\
\hline & Stage terrain (3 jours). & $\begin{array}{l}\text { Familiarisation avec le milieu rural à partir de la visite de sites } \\
\text { présélectionnés révélateurs de la diversité des contextes; initiation } \\
\text { à la lecture des paysages. }\end{array}$ \\
\hline A & $\begin{array}{l}\text { Exercice } 1: \text { Lecture du } \\
\text { paysage et identification } \\
\text { préliminaire des enjeux ( } 2 \\
\text { semaines). }\end{array}$ & $\begin{array}{l}\text { Lecture du paysage de l'ensemble du territoire d'étude conduisant à } \\
\text { l'identification d'une liste préliminaire d'enjeux. Cet exercice est } \\
\text { suivi d'une rencontre avec les élus et intervenants du milieu pour } \\
\text { échanger sur les enjeux mutuellement perçus. C'est sur la base de } \\
\text { cet échange, identification, par chaque équipe, de l'enjeu traité au } \\
\text { cours de la session. }\end{array}$ \\
\hline C & $\begin{array}{l}\text { Exercice } 2: \text { Diagnostic } \\
\text { paysager relatif à l'enjeu } \\
\text { retenu (5 semaines). }\end{array}$ & $\begin{array}{l}\text { À partir des bases de données et des documents fournis par la } \\
\text { collectivité, de relevés de terrain, d'échanges ponctuels avec des } \\
\text { personnes ressources (citoyens, professionnels, etc.), réalisation } \\
\text { d'un diagnostic relatif à l'enjeu considéré (ex.: analyse de la } \\
\text { dynamique des paysages, relevés visuels, caractérisation } \\
\text { patrimoniale, etc.). Production d'un rapport de diagnostic. }\end{array}$ \\
\hline
\end{tabular}




\begin{tabular}{|c|c|c|}
\hline 0 & $\begin{array}{l}\text { Exercice } 3: \text { Prise de } \\
\text { position et orientation } \\
\text { privilégiée (2 semaines). }\end{array}$ & $\begin{array}{l}\text { Sur la base de l'exercice } 2 \text { et d'un relevé des précédents, prise de } \\
\text { position quant à la nature de l'orientation privilégiée et des } \\
\text { aménagements considérés. Première présentation devant un jury } \\
\text { restreint composé de professeurs, de professionnels et de } \\
\text { représentants de la collectivité pour valider le diagnostic et } \\
\text { l'orientation privilégiée. }\end{array}$ \\
\hline 0 & $\begin{array}{l}\text { Exercice 4: Propositions } \\
\text { d'aménagement } \\
\text { semaines). }\end{array}$ & $\begin{array}{l}\text { Élaboration de propositions d'intervention. Présentation de } \\
\text { l'ensemble des résultats devant un jury composé de professeurs, de } \\
\text { professionnels, d'élus et de citoyens. Réalisation de deux affiches } \\
\text { format A0 (une pour le diagnostic, une pour les propositions) pour } \\
\text { l'exposition à tenir en région. }\end{array}$ \\
\hline 0 & $\begin{array}{l}\begin{array}{l}\text { Retour à la collectivité } \\
\text { sous } \\
\text { appropriables. }\end{array} \\
\text { formes }\end{array}$ & $\begin{array}{l}\text { Se fait le plus souvent en deux temps et sous deux formes: } \\
\text { - présentation, par quelques équipes, devant le conseil municipal } \\
\text { (janvier suivant l'atelier); } \\
\text { - exposition des travaux de l'ensemble des équipes (vernissage et } \\
\text { conférence de presse) en région (municipalité collaboratrice). }\end{array}$ \\
\hline
\end{tabular}

11 Au niveau de la recherche ensuite, un regard rétrospectif révèle que l'atelier peut s'avérer une plate-forme d'échanges et d'enrichissement mutuel entre recherche, formation et pratique. Jouant le rôle tantôt de laboratoire de validation des résultats de recherche, tantôt d'accompagnateur de recherches en cours, tantôt encore de lieu de développement de connaissances, l'atelier est, à travers les collaborations et restitutions des travaux réalisés (présentations publiques, expositions des travaux, sites internet, etc.), une voie privilégiée de transfert des connaissances. En retour, il constitue aussi une mise à l'épreuve de celles-ci. D'une part, les étudiants adoptent le plus souvent une position critique qui questionne la validité des propositions issues de la recherche. D'autre part, l'appropriation de ces connaissances par les collectivités génère régulièrement de nouvelles questions de recherche.

Cela dit, les difficultés et limites posées par un tel atelier demeurent nombreuses (Domon, 2017b). Pour l'étudiant d'abord, elles tiennent aux changements d'échelle (du site à la région), de matériaux (du minéral et de l'horticole à l'agricole) et de la nature de la démarche (design d'espaces publics urbains vs idéation/accompagnement de démarches des communautés rurales). Plus encore, elles découlent du passage d'enseignements où l'emphase porte sur la dimension création/design à un atelier où, dans la première partie à tout le moins, l'accent est mis sur la dimension analytique, voire scientifique. Sur ce plan, bien que certains aient souligné la nécessité de dépasser la dichotomie art/sciences au risque de perdre de vue une des spécificités de l'architecture de paysage (Gazvoda, 2002), l'expérience révèle que, dans les faits, seule une minorité d'étudiants réussit à présenter un même niveau d'excellence dans ces deux dimensions de la profession. 
13 Pour l'acquisition de compétences ensuite, force est d'admettre que le calendrier académique a pour effet d'imposer des contraintes majeures. Limité à un seul trimestre, l'atelier demeure essentiellement un lieu de familiarisation aux grands paysages et ne peut ainsi permettre l'approfondissement ni des dimensions politiques de l'aménagement, ni des enjeux identifiés, ni même d'accompagner les collectivités dans la durée. Si les uns peuvent déplorer, au terme de chacune des éditions, le caractère inachevé des propositions au plan du design, l'absence de connaissance suffisante des forces motrices, soit des forces économiques, politiques et législatives (Bürgi et al., 2009) qui agissent sur les paysages, ressort comme particulièrement limitative en regard de la valeur des propositions. Aussi, en absence de formation suffisante, les analyses, réflexions et propositions sont-elles condamnées à porter sur les seules formes visibles ("Shallow form», Van Damme, 2015) plutôt que sur les processus qui agissent sur elles. Du coup, le travail ne rejoint pas ce qui fonde et transforme le visible (Briffaud, 2008).

14 C'est notamment en raison de ces limites qu'a été conçue et mise en place une maîtrise professionnelle en architecture de paysage à l'automne 2012.

\section{La maîtrise en architecture de paysage comme seconde réponse à la demande sociale en paysage}

Deux principaux constats étaient à la base de cette nouvelle maîtrise professionnelle. D'une part, il s'agissait de reconnaître que, à l'instar d'autres disciplines connexes (architecture, urbanisme, etc.), la pratique professionnelle s'était à ce point développée et complexifiée qu'une seule formation de premier cycle ne permettait plus d'acquérir les compétences nécessaires. D'autre part, il s'agissait également de reconnaître que, tout en se complexifiant, la pratique s'était aussi diversifiée rendant de plus en plus difficile pour un même individu de couvrir l'ensemble des formes qu'elle pouvait revêtir.

16 Le nouveau programme mettait ainsi de l'avant deux parcours, "Design avancé » et «Grand paysage » qui visaient à permettre, par-delà un tronc commun portant sur les dimensions théoriques (ex. : cours de théories du paysage) et méthodologiques (ex. : atelier de caractérisation des paysages), d'acquérir des compétences davantage mobilisées par certaines formes de pratique (tableau 2). De permettre donc une certaine forme de spécialisation. 
Tableau 2. Les deux parcours de la maîtrise en architecture de paysage

\begin{tabular}{|l|l|}
\hline Design avancé & Grand paysage \\
\hline $\begin{array}{l}\text { Atelier : « Caractérisation des paysages » } \\
\text { Cours : « Théorie du paysage », « Méthodologie de projet de paysage », « Pratiques des disciplines } \\
\text { de l'aménagement », séminaire de fin d'études }\end{array}$ \\
$\begin{array}{l}\text { Atelier « Design avancé » } \\
\text { Construction avancée, etc. }\end{array}$ & $\begin{array}{l}\text { Atelier « Grand paysage » } \\
\text { Écologie du paysage, etc. }\end{array}$ \\
\hline Projet de fin d'études en design avancé & Projet de fin d'études en grands paysages \\
\hline
\end{tabular}

Ces deux parcours ("Design avancé » et « Grand paysage ») partageaient un tronc commun et se distinguaient à travers des activités spécifiques.

17 Dans le cas du parcours "Grand paysage ", le programme prévoyait de permettre à l'étudiant d'acquérir ces compétences par le biais de trois groupes d'activités :

- l'inscription à certains cours à option en lien avec les grands paysages (ex. : écologie du paysage, espaces naturels, etc.) ;

- la participation à l'atelier «Grand paysage » dont l'objectif était de permettre la poursuite des collaborations amorcées dans l'atelier de baccalauréat mais en mettant l'accent sur les forces motrices et les processus qui façonnent les paysages ;

- la réalisation d'un projet de fin d'études pour lequel les directives étaient suffisamment souples pour s'accommoder, selon les contextes et problématiques retenus, de projets mettant l'accent tantôt sur le développement ou la mise en application de compétences relatives aux programmes et politiques d'aménagement des paysages, tantôt sur les outils et méthodes de caractérisation des paysages, tantôt encore sur des propositions d'intervention physico-spatiale.

18 Cette approche de la formation, guidée par une lecture plurielle de la pratique professionnelle et par l'ouverture au développement de nouvelles compétences, allait se heurter, lors du processus d'accréditation professionnelle du programme, tenu à l'automne 2015, à une autre vision de la formation et de la profession, celle portée par l'AAPC.

Ainsi, du rapport d'accréditation du programme (AAPC, 2015b), il ressort que :

- le «cœur» de la formation et de la profession repose sur un ensemble de compétences à prédominance technique nécessaires à la pratique du design : compétences en matériaux, en dessins techniques et devis, en assemblage, en informatique appliquée, etc. ${ }^{3}$;

- tous les étudiants doivent acquérir les mêmes compétences ;

- le travail de fin d'études doit être l'occasion de démontrer la capacité de l'étudiant à mettre en application ses compétences en matière d'intervention physico-spatiale.

20 Aussi, dans la foulée de ce rapport, une demande de modification de programme allait devoir être déposée, le texte stipulant que «[...] le programme de maitrise doit offrir à tous les étudiants, y compris les étudiants gradués de notre baccalauréat, une formation incluant les connaissances, méthodes et approches de travail appliquées à l'ensemble des contextes, échelles et domaines d'intervention en architecture de 
paysage, conformément à la liste ci-dessus. Pour satisfaire aux normes d'agrément, les deux concentrations actuellement offertes doivent être abolies. » (Anonyme, 2016, p. 3).

La vision de la formation portée par L'AAPC ressort donc comme plus restrictive et plus instrumentale que celle à l'origine du programme professionnel de second cycle initialement mis en place. Davantage encore, lorsque mise en relation avec la définition du champ disciplinaire proposée par Williams (2014) et citée précédemment, cette vision soulève un certain nombre d'enjeux sur ce que doit être la formation, voire sur ce qu'est l'architecture de paysage.

\section{Discussion : des visions différentes sur des enjeux au cœur de la formation et de la profession}

22 Au Québec, la formation en paysage relève donc essentiellement des programmes en architecture de paysage de l'École d'urbanisme et d'architecture de paysage de l'université de Montréal ${ }^{4}$. Or, si les différents acteurs semblent partager des définitions semblables de la profession ${ }^{5}$, l'exercice mené dans les pages qui précèdent révèle qu'en pratique des divergences significatives existent en matière de formation et de champ d'action. De fait, la portée et les limites de la profession ont suscité une abondante littérature au fil des ans (Marusic, 2002 ; Feng et al., 2010; Gobster et al., 2010 ; etc.). Aussi, au sein de celle-ci, trois enjeux ressortent de manière récurrente. Les paragraphes qui suivent les abordent en prenant pour base l'exemple des grands paysages.

\section{La diversité des pratiques}

23 Animée par des valeurs environnementales, sociales et esthétiques (Thompson, 2002), l'architecture de paysage aurait pour finalité la création, l'amélioration, le maintien et la protection de places fonctionnelles, esthétiques, plaisantes, signifiantes, soutenables et appropriées pour les besoins et objectifs des individus (ECLAS, 2010), et ce dans tous les types de milieux et à toutes les échelles (Gazvoda, 2002 ; Williams 2014). Aussi, un consensus se dégage-t-il quant au fait qu'un tel projet commande une large gamme de pratiques (ECLAS, 2010) relevant certes de la planification, de la conception et de la gestion des paysages (Herlin et Stiles, 2016 ; Meijering et al., 2015 ; ECLAS, 2010, etc.) mais aussi de l'évaluation scientifique (Donadieu et Aggéri, 2011), de la médiation (Donadieu, 2009) et de l'accompagnement (Paquin et al., 2014). Si, d'aucuns peuvent mettre en doute la capacité d'une discipline unique à réaliser un projet aussi ambitieux (voir ci-après), Sharky relève à cet égard que "(r)ight now landscape architecture claims to be able to do everything (Sharky, 2016, p. 208).

Sur ce plan, la position de l'AAPC (AAPC 2015b) se démarque à un point tel qu'elle laisse poindre l'existence d'une "vision canadienne" de l'architecture de paysage. En insistant sur l'importance des connaissances techniques, au détriment de celles portant sur les forces (économiques, politiques, culturelles, etc.), qui façonnent les paysages et sur lesquelles reposent les activités de planification, de gestion et de conservation, les normes de l'AAPC ont inévitablement pour effet de tendre à centrer la formation dans un type de pratique spécifique (celle du design), exercé dans un contexte spécifique (contexte urbain ou périurbain) et à une échelle spécifique (échelle du site, du lot ou de 
la municipalité). Même si, comme relevé en introduction, les préoccupations pressantes en matière de paysage se traitent mieux à l'échelle régionale, «[...] notre profession n'envisage que l'échelle locale [...] (l)es processus d'agrément professionnel sont axés sur la conception et la construction à l'échelle d'un site et reconnaissent à peine une pratique à plus vaste échelle » (Trottier, 2017, p. 77).

D'aucuns pourront penser que, dans une volonté de prendre en compte les spécificités régionales (AAPC, 2015a), cette place quasi exclusive accordée à la conception à l'échelle locale résulterait de certaines caractéristiques de la demande en paysage propres au Canada. Or, tel ne semble pas le cas, au Québec du moins. D'une part, l'analyse historique (période 1977-2012) des démarches pour la prise en compte du paysage dans les pratiques d'aménagement (Domon, 2017a) révèle l'ampleur de celles qui empruntent des voies autres que la conception d'espaces. D'autre part, aussi partiel et partial qu'il soit, le relevé des initiatives en grands paysages, ayant donné lieu à une collaboration (effective ou sollicitée) avec la chaire en paysage et environnement de l'université de Montréal, au cours de l'année où a été menée l'accréditation du programme de maitrise (voir tableau 3), aide à mieux saisir l'ampleur et la nature des besoins exprimés par les instances locales et régionales. Sauf exception, ces besoins relèvent non pas de la conception d'espaces mais plutôt de la caractérisation, de l'accompagnement de démarches (démarches de création de chartes, d'obtention de statuts, etc.) et de la gestion (gestion des paysages agricoles). C'était pour répondre à ces besoins et tenir compte des particularités du contexte québécois qu'avait initialement été pensé et conçu le parcours « Grand Paysage ». Davantage qu'aux seules compétences techniques sur lesquelles insiste l'AAPC, les besoins exprimés par les collectivités renvoient donc à cette autre catégorie de compétences qui, selon l'European Council of Landscape Architecture Schools (ECLAS), seraient au cœur de l'architecture de paysage à savoir: "(an) holistic knowledge and understanding of the nature of landscape and the ways in which it is perceived in time and space, and the pressures and driving forces to which landscapes are subjected " (ECLAS, 2010, p. 13).

Ainsi, comme « les organes directeurs de l'AAPC et de l'American Society of Landscape Architects (ASLA) font obstacle à l'agrément et à la reconnaissance des professionnels émergents qui pratiquent des formes alternatives d'architecture paysagère " (Trottier, 2017, p. 77), cette vision plus restrictive de la pratique laisse en plan une gamme significative de besoins exprimés par les instances locales et régionales.

Tableau 3. Initiatives en grand paysage ayant donné lieu à une collaboration (effective ou sollicitée) avec la chaire en paysage et environnement de l'université de Montréal au cours de l'année de l'accréditation (2015-2016)

\begin{tabular}{|l|l|}
\hline Porteur de l'initiative & Nature de la démarche \\
\hline Ville de Gaspé & Élaboration d'une charte des paysages \\
\hline MRC La Côte de Gaspé & Démarche pour l'obtention du statut de paysage humanisé \\
\hline MRC d'Argenteuil & Diagnostic paysage, Vallée de la Harrington \\
\hline MRC des Appalaches & $\begin{array}{l}\text { Diagnostic paysager et amorce d'une démarche collective de } \\
\text { mise en valeur }\end{array}$ \\
\hline
\end{tabular}




\begin{tabular}{|l|l|}
\hline MRC Brome-Missisquoi & Gestion des paysages agricoles en contexte de déprise \\
\hline MRC de Memphrémagog & $\begin{array}{l}\text { Mise en place d'un observatoire photographique et gestion des } \\
\text { paysages agricoles en contexte de déprise }\end{array}$ \\
\hline MRC Bécancour & $\begin{array}{l}\text { Diagnostic paysager et amorce d'une démarche collective de } \\
\text { mise en valeur }\end{array}$ \\
\hline $\begin{array}{l}\text { Municipalité de Saint-Georges- } \\
\text { de-Clarenceville }\end{array}$ & $\begin{array}{l}\text { Sollicitation et collaboration à l'atelier « Espace régional » } \\
\text { paysage niveau baccalauréat }\end{array}$ \\
\hline MRC de Marguerite-D'Youville & Exposition des travaux de l'atelier «Espace régional » \\
\hline MRC Pays d'en Haut & Caractérisation du patrimoine paysager \\
\hline $\begin{array}{l}\text { MRC Pays d'en Haut } \\
\text { MRC de la Rivière-du-Nord } \\
\text { MRC d'Antoine Labelle des Laurentides }\end{array}$ & $\begin{array}{l}\text { Amorce d'une démarche d'obtention du statut de paysage } \\
\text { culturel patrimonial }\end{array}$ \\
\hline MRC Les Moulins & $\begin{array}{l}\text { Intégration d'un développement résidentiel au paysage agricole } \\
\text { existant. }\end{array}$ \\
\hline
\end{tabular}

\section{Le niveau de spécialisation}

De l'avis général, l'architecture de paysage serait donc une discipline capable de résoudre des problèmes complexes en faisant appel à un large éventail de connaissances pluridisciplinaires des sciences, naturelles et humaines, de même qu'à la créativité (Gazvoda, 2002; Thompson, 2002 ; Feng et al., 2010, etc.). Or, comment maintenir cette capacité alors que certains domaines au sein desquels s'alimentent tout particulièrement les architectes paysagistes (écologie, génie, etc.) se sont développés au point de créer des "sous-disciplines » (écologie animale, écologie végétale, etc.) et que sont apparus de nouveaux problèmes complexes (ex.: changements globaux)? Si, comme déjà relevé, «[...] landscape architecture claims to be able to do everything " (Sharky, 2016, p. 208), certains reconnaissent en contrepartie que «[...] landscape architects cannot be specialists in all [...] » (Herlin et Stiles, 2016, p. 179) et que "nous devons perdre cette croyance complaisante selon laquelle les architectes paysagistes sont intrinsèquement qualifiés pour pratiquer à toutes les échelles " (Trottier, 2017, p. 77). La question d'une éventuelle spécialisation et, le cas échéant, du niveau de spécialisation souhaitable est ainsi un second thème récurrent au sein de la littérature. Sur ce plan, alors que certains reconnaîtront la pertinence, voire la nécessité d'une certaine spécialisation (Herlin et Stiles, 2016) au point de reconnaître l'existence de différents métiers (Donadieu, 2009), d'autres y voient plutôt le risque de perdre ce qui constituerait le cœur de la profession (Gazvoda, 2002 ; Marušič, 2002, etc.), soit d'être une discipline de synthèse.

28 À cet égard, la «vision canadienne » telle que véhiculée par l'AAPC paraît particulièrement claire puisque l'importance accordée à la pratique du design à 
l'échelle locale sous-tend la promotion d'une certaine forme de spécialisation. Cela dit, cette position soulève des tensions à deux niveaux.

Premièrement, à la lumière de ce qui a été dit précédemment au regard de l'ampleur et de la nature des besoins des collectivités locales et régionales en matière de paysage, il est inévitable que des professionnels soient appelés à exercer des pratiques ne relevant pas du design : développement d'outils de caractérisation, réalisation de diagnostics, accompagnement, médiation, etc. Or, pour ne prendre que cette dernière pour exemple, il est clair que « les architectes paysagistes ne sont pas formés pour être des arbitres entre les acteurs publics et de la production des paysages » (Donadieu, 2009, p. 54).

D'autre part, tout en faisant du design le cœur de la pratique, l'AAPC insiste sur l'importance que tous acquièrent les mêmes compétences. Du coup, se démarquant d'une certaine tendance internationale (Herlin et Stiles, 2016), elle vient freiner la spécialisation au sein des programmes de formation et poser que les futurs professionnels pourront agir dans tous les types de milieux (urbain, périurbain, rural, naturel) et à toutes les échelles. Pourtant, le niveau de mobilisation des connaissances varie considérablement d'un contexte à l'autre. Par exemple, alors que les interventions dans les grands paysages à prédominance agricole pourront s'accommoder de compétences limitées dans les domaines de la «construction avancée » et du "dessin technique », elles nécessitent une connaissance et une compréhension très fines tant de la législation que des programmes, des politiques et des pratiques agricoles. Sur ce plan, l'expérience cumulée dans l'atelier «Espace régional » laisse peu de doute quant au fait qu'en absence d'une telle connaissance, la crédibilité du professionnel en architecture de paysage sera mise à mal. Ainsi, si intéressantes soient-elles aux plans esthétique et formel, des propositions sur l'implantation de bandes riveraines, sur les réaffectations des bâtis patrimoniaux agricoles, sur les aménagements des abords de ferme ou sur la mise en valeur des cours d'eau agricoles auront peu de crédibilité si elles ne témoignent pas d'une parfaite maitrise du contexte d'exploitation. Certes, le professionnel en architecture de paysage n'opérera pas seul et sera appelé à travailler le plus souvent en contexte multidisciplinaire. Aussi, la question demeure-t-elle : une formation peut-elle conduire à un niveau de compétences suffisant pour qu'un même individu puisse échanger « d'égal à égal » tantôt avec des agronomes, des biologistes de la faune et des ingénieurs hydrauliques, tantôt avec des architectes, des urbanistes, et des ingénieurs civils, tantôt encore avec des biologistes de la conservation, des ingénieurs forestiers, des spécialistes du tourisme, etc. En définitive, la question de la spécialisation au sein de la formation demeure entière.

\section{La place de la recherche dans un programme professionnel}

31 L'image du médecin est fréquemment utilisée pour expliquer le rôle de l'architecte paysagiste (Thompson, 2002; Donadieu, 2009; Brown et Corry, 2011). Ainsi, "(l)ike doctors, designers could analyse a site or a situation, diagnose its problems and prescribe a solution" (Thompson, 2002, p. 83). En regard de cette comparaison, Brown et Corry (2011) apportent toutefois une nuance importante. Alors que la médecine est, au fil des années, devenue une discipline académique dont les recommandations reposent sur des 
évidences, des faits, des expérimentations et des savoirs stabilisés, "much of contemporary practice in landscape architecture is still based on beliefs rather than facts" (Brown et Corry, 2011, p. 327). Ces propos rejoignent ceux des nombreux auteurs (Palea et al., 2012 ; Van den Brink et Bruns, 2012 ; Lenzholder et al., 2013 ; Gobster, 2014 ; Van Damme, 2015, etc.) qui, ces dernières années, ont plaidé pour une place accrue de la recherche au sein de la discipline : "If landscape architecture is to reach greater academic distinction, it is important that more research is conducted to create a sound evidence base that helps to justify landscape planning, design, and management decisions. » (Meijering et al., 2015, p. 86.) Cette exhortation à la recherche n'est pas nouvelle puisque Gobster nous rappelait que «Landscape Journal (LJ) was launched in 1982 in response to the increasing perception of educators in landscape architecture that the profession, to grow as a discipline, must take responsibility for generating its own knowledge base of research and other scholarly inquiry » (Gobster, 2010).

Compte tenu de son caractère professionnel, la discipline soulève par ailleurs inévitablement la question suivante: "Le praticien paysagiste peut-il être en même temps chercheur?» (Donadieu, 2009, p. 105) ; question complexe, source de tension au sein des départements universitaires offrant des programmes professionnels, et qui renverrait à «deux professions différentes qui requièrent des compétences et des diplômes distincts" (ibid.). Misant sur le rapprochement de l'une et de l'autre, le programme initial de maitrise en architecture de paysage restait ouvert sur le fait que le projet de fin d'études puisse porter sur l'exploration, l'expérimentation et la production de connaissances en amont de l'intervention. Sur ce plan, l'AAPC invitait plutôt à recentrer le travail dirigé sur la pratique et sur l'intervention physico-spatiale en notant que « la raison d'être des projets de thèse et les résultats attendus doivent être clarifiés pour s'assurer que tous les diplômés maitrisent les compétences fondamentales de la discipline dans les études et les applications complexes » (AAPC, 2015c, p. 2). Du coup, cette recommandation allait amener l'École d'urbanisme et d'architecture de paysage à développer puis à mettre en place, à l'automne 2017, un nouveau programme de maitrise non accréditée ${ }^{6}$ et ainsi à reconduire la division entre formation professionnelle et formation à la recherche.

\section{Conclusion}

Le processus d'accréditation a pour intérêt de susciter une profonde réflexion sur la nature et la finalité d'une formation professionnelle en architecture de paysage, voire sur la discipline en elle-même. Force est de constater qu'à ce jour cette formation n'est pas en mesure de combler un pan important de la demande sociale en paysage qui se manifeste au Québec tant chez les acteurs publics (municipalités, ministères, etc.) que dans les collectivités. Outre le fait que cette demande demeure relativement récente et souvent mal évaluée, voire sous-estimée, trois principaux facteurs peuvent être évoqués pour comprendre cet état de fait.

Alors que, comme le soulignait Sharky (2016), l'architecture de paysage a historiquement soutenu " to be able to do everything " en matière d'intervention sur les paysages, les domaines d'action se sont à ce point diversifiés et spécialisés qu'une telle position paraît aujourd'hui difficilement soutenable. Sur ce plan, relevons à nouveau que les normes d'accréditation supportées par l'AAPC ont fortement recentré la formation sur la seule pratique du design à l'échelle locale. Signalons toutefois, à la 
lumière de différents textes sur la formation et l'évolution de la pratique en Europe (ECLAS, 2010; Herlin et Stiles, 2016) que ce recentrement ne paraît pas inhérent à la discipline et qu'il résulterait plutôt d'une certaine spécificité (canadienne) de celle-ci ; spécificité qui se trouve en porte-à-faux avec les réalisations de pionniers nordaméricains qui, tel Ian McHarg, ont mené des travaux marquants en planification régionale des paysages.

Ensuite, et dans ces circonstances, il importe de relever à nouveau que la formation en paysage offerte au Québec se résume aux seuls programmes en architecture de paysage de l'université de Montréal. La situation contraste donc fortement avec celle rencontrée en Europe où quelque 95 programmes, dont certains plus spécialisés (Herlin et Stiles, 2016), sont proposés.

Enfin, force est de reconnaître que pour le nouveau professionnel en paysage désirant travailler en milieu rural et à l'échelle régionale, l'employabilité demeure un défi. Ce défi est d'autant plus considérable qu'au Québec, en raison de l'importance des dimensions légale et réglementaire portées par la loi sur l'aménagement et l'urbanisme, une formation en urbanisme demeure souvent un passage quasi obligé pour tout professionnel voulant œuvrer en aménagement du territoire.

Cela dit, la demande sociale en grand paysage et la diversité des pratiques qu'elle commande demeurent telles qu'elles gagneront à trouver davantage écho dans la formation. Un premier pas a été franchi avec l'instauration du programme de second cycle non accrédité, la maîtrise " Ville, Territoire, Paysage ", qui laisse place à une large gamme d'approches pour l'étude et l'intervention sur les paysages. Un second pourrait être fait en ne limitant pas systématiquement le travail de fin d'études, dernier atelier pédagogique de la formation, à la seule intervention physico-spatiale. Enfin, ultimement, il faudra en arriver à faire en sorte que, comme le propose Trottier (2017), le processus d'accréditation canadien s'ouvre aux « formes alternatives d'architecture paysagère ».

L'auteur remercie les deux évaluateurs anonymes qui ont commenté une version préliminaire et ont ainsi permis de préciser et de bonifier le contenu du présent texte.

\section{BIBLIOGRAPHIE}

Anonyme, « Demande de révision du programme, Maîtrise en architecture de paysage (MAP) », École d'urbanisme et d'architecture de paysage, université de Montréal, 2015, 14 p.

Association des architectes paysagistes du Canada (AAPC), « Manuel des normes et procédures pour l'agrément des programmes d'études canadiens en architecture de paysage », 2015a, $38 \mathrm{p}$. + annexes, URL : http://www.aapc-csla.ca/sites/csla-aapc.ca/files/accreditation/LAAC Standards and Procedures 2014 Draft_2.FR final February 2015.pdf.

Association des architectes paysagistes du Canada (AAPC), « Master of Landscape architecture, University of Montreal, Accreditation Report », texte inédit, non publié, 2015b, 29p. 
Association des architectes paysagistes du Canada (AAPC), « Maîtrise en architecture de paysage de l'université de Montréal. Résumé de la décision et des recommandations en matière d'agrément ", texte inédit, non publié, 2015c, 3 p.

Briffaud, S., « Le projet entre recherche et création », texte de conférence, Arthous, octobre 2008. Bureau, L, « Des paysages, des idées et des hommes : le projet collectif de Charlevoix », Cahiers de géographie du Québec, vol. 21, n53-53, 1977, p. 187-219.

Brown, R.D. et R.C. Corry, « Evidence-Based Landscape Architecture : The Maturing of a Profession ", Landscape and Urban Planning, vol. 100, 2011, p. 327-329.

Bürgi, M., Straub, A., Gimmi, U. et Salzmann, D., « The recent landscape history of Limpach valley, Switzerland : considering three empirical hypotheses on driving forces of landscape change ", Landscape Ecology, vol. 25, nº 2, 2009, p. 287-297.

Couillard, M.-A., « Les paysages de Clarenceville étudiés à l'université de Montréal », Coup d'œil.info, 10 février 2016, p. 3.

Domon, G., « La prise en compte du paysage dans l'aménagement des milieux ruraux québécois : un regard sur les 35 premières années ", dans Chiasso, G., Ependa, A., Jean, B. et Leblanc, P. (dir.), La Ruralité au Québec depuis les États généraux du monde rural (1991), Rouyn-Noranda, Chaire Desjardins en développement des petites collectivités, université du Québec en AbitibiTémiscamingue, 2017a, p. 25-44.

Domon, G., « Le grand paysage : les défis de la formation », Lansdscapes|Paysages, vol. 19, $\mathrm{n}^{\circ}$ 1, 2017b, p. 43.

Domon, G., Froment, J., Ruiz, J. et Vouligny, É, « Les paysages de l'ordinaire, révéler, créer, infléchir - Dix projets de mise en valeur des paysages du canton de Kildare », École d'architecture de paysage et chaire en paysage et environnement, 2006, $109 \mathrm{p}$.

Donadieu, P. et Aggéri, A , «Quatre postures de valorisation et d'évaluation des paysages dans les régions de montagne : le designer, le planificateur, le gestionnaire et le scientifique ", Projets de paysage, $\mathrm{n}^{\circ}$ 6, juillet 2011, URL : http://www.projetsdepaysage.fr/fr/

quatre_postures_de_valorisation_et_d_evaluation_des_paysages_dans_les_regions_de_montagne_le_designer_le_planificateur_le_ges Donadieu, P., Les Paysagistes, Arles/Versailles, Actes sud/École nationale supérieure de Paysage, $2009,139 \mathrm{p}$.

European Council of Landscape Architecture Schools (ECLAS), « Tuning Landscape Architecture Education in Europe », version 26, 2010, 58 p., URL : http://www.unideusto.org/tuningeu/ images/stories/Summary_of_outcomes_TN/

ECLAS_Guidance_on_Landscape_Architecture_Education.pdf.

Feng, L., Zhao, X. et Liu, Y., « Discussion and Consideration on Teaching Reform of Landscape Architecture », dans « 2010 International Conference on Education and Management Technology », 2010, p. 138-141.

Gazvoda, D., " Characteristics of modern landscape architecture and its education », Landscape and Urban Planning, vol. 60, 2002, p. 117-133.

Gobster, P. H., « (Text) Mining the LANDscape : Themes and trends over 40 years of Landscape and Urban Planning », Landscape and Urban Planning, vol. 126, 2014, p. 21-30.

Gobster, P., Iverson Nassauer, J. et Nadenicek, D.J., « Landscape Journal and Scholarship in Landscape Architecture The Next 25 Years », Landscape Journal, vol. 29, 2010, p. 52-70. 
Herlin, I.S. et Stiles, R., « The European Landscape Convention in Landscape Architecture Education », Jorgensen, K., Clemetsen, M., Halvorsen Thorén, A.-K., et Richardon, T. (ed.), Mainstreaming Landscape Through the European Landscape Convention, Rouledge, London, 2016, p. 175-186.

Lacasse, O., «L'amorce du projet de paysage local. Étude de cas : Saint-Étienne-de-Bolton », mémoire de maîtrise, université de Montréal, 1995.

Lenzholder, S., Duchhart, I. et Koh, J., " 'Research through designing' in landscape architecture ", Landscape and Urban Planning, vol. 113, 2013, p. 120-127.

Marušič, I., " Some observations regarding the education of landscapearchitects for the 21st century », Landscape and Urban Planning, vol. 60, 2002, p. 95-103.

McHarg, I., Design with Nature, New-York, Natural History Press, 1969, 198 p.

Meijering, J. V., Tobi, H., van den Brink, A., Morris, F. et Bruns, D., « Exploring Research Priorities in Landscape Architecture : An international Delphi study », Landscape and Urban Planning, vol. 137, 2015, p. 85-94.

Milburn, L.-A. S. et Brown, R. D., « The Relationship between Research and Design in Landscape Architecture ", Landscape and Urban Planning, vol. 64, 2003, p. 47-66.

Palea, A., Ciobanu, G., et Kilyeni, A., « Educational Skills in Training Landscape Architecture Students : Developing Communication Skills », Procedia - Social and Behavioral Sciences, vol. 46, 2012, p. 4672-4677.

Paquin, C., Domon, G.et Ruiz, J., « Vers des scénarios d'aménagements multifonctionnels en zone d'intensification agricole : portée et limites des politiques et pratiques québécoises » dans Ruiz, J. et Domon, G (dir.), Agriculture et Paysage. Aménager autrement les territoires ruraux, Montréal, Presses de l'université de Montréal, 2014, p. 307-325.

Pernet, A., Le Grand Paysage en projet, Les Acacias, MétisPresses, 2014, 318 p.

Raveneau, J. « Analyse morphologique, classification et protection des paysages : le cas de Charlevoix ", Cahiers de géographie du Québec, vol. 21, n 53-54, 1977, p. 135-186.

Sharky, L., Thinking about Landscape architecture. Principles of a Design Profession for the 21th Century, Routledge, London, 2016, 220 p.

Thompson, I. H., « Ecology, community and delight : a trivalent approach to landscape education », Landscape and Urban Planning, vol. 60, 2002, p. 81-93.

Trottier, J., « Une échelle réduite », Landscapes|Paysages, vol. 19, n 4, 2017, p. 77.

Van Damme, S., « Back to the future : merging landscape concepts of the 1980s into the truly holistic framework of tomorrow ", Journal of landscape Architecture, vol. 10, nº 3, 2015, p. 52-67.

Van den Brink, A. et Bruns, D., « Strategies for Enhancing Landscape Architecture Research », Landscape Research, vol. 39, $\mathrm{n}^{\circ}$ 1, 2012, p. 7-20.

Weller, R. «A call for action ", in Landcscape Architecture Foundation (ed.), The New Landscape Declaration. A call to action for the twenty-first century, Los Angeles, Rare Bird Books, 2017, p. 5-11. Williams, R., L'Architecture de paysage au Canada, Montréal, Presses de l'université de Montréal, $2014,672 \mathrm{p}$. 


\section{NOTES}

1. Les termes "révolution tranquille " se réfèrent à une période, les années 1960 , au cours de laquelle le Québec a connu d'importants changements sociaux, notamment une forte diminution du rôle de l'Église dans les affaires publiques et une intervention importante de l'État dans diverses sphères de la société.

2. Aujourd'hui École d'urbanisme et d'architecture de paysage.

3. Voir notamment ce passage qui couvre l'examen des cours offerts dans le programme: «A. The following core program elements shall be included [...] in order to permit the achievement of the primary objective, the education of students for the professional practice of Landscape Architecture. [...]

1. The history and theory of Landscape Architecture [...].

2. The theory and practice of design in Landscape Architecture, including :

- principles of design ;

- environmental and socio-cultural constraints and requirements;

- graphic, written and oral communication of design analysis, concepts, and solutions ;

- preparation of plans at the analytical, conceptual, and design stages. Project scales should vary as should the context in which they are undertaken (urban, rural, wilderness, etc.); and

- working drawings and specification writing. ॥ (AAPC, 2015b, p. 9.)

4. Soulignons que, outre des cours portant sur des dimensions spécifiques telles que l'écologie du paysage ou la géomorphologie et des cours où le paysage est une des composantes traitées (ex. : développement rural DEV 78185 offert à l'UQAR), des cours portant spécifiquement sur le sujet sont offerts à l'université Laval (paysage: analyse, protection et mise en valeur GGR 3400 ; paysage : art, science et politique GGR 7010) et à l'université du Québec à Trois-Rivières (analyse du paysage GEO 1126). Cela dit, les programmes en architecture de paysage demeurent les seuls dont le diplôme porte le terme paysage.

5. Rejoignant celle de Williams, l'AAPC définit ainsi la profession : "Qu'est-ce que l'architecture de paysage ? L'architecture de paysage concerne la conception, la planification, la gestion et la préservation du territoire. » (URL : http://www.aapc-csla.ca/society/lassociation, consultée le 28 avril 2018.)

6. Ce programme «[...] propose des regards croisés sur les enjeux contemporains de la transformation des villes, territoires et paysages d'ici et d'ailleurs. Résolument orientée vers la multidisciplinarité, la formation prend appui et encourage le développement de pratiques et savoirs innovants à la croisée de l'urbanisme, de l'architecture de paysage, du développement régional, de l'aménagement et $\mathrm{du}$ développement $\mathrm{du}$ territoire. » URL: https:// admission.umontreal.ca/programmes/maitrise-en-amenagement-option-ville-territoire-

paysage/.

\section{RÉSUMÉS}

$\mathrm{Au}$ Québec, la formation en paysage présente certaines singularités. Ainsi, elle tient aux seuls programmes offerts à l'université de Montréal. De même, le programme professionnel de second cycle est soumis à l'accréditation de l'Association des architectes paysagistes du Canada (AAPC). En milieu universitaire, cette situation est singulière, le programme découlant non seulement de la vision de ses responsables mais aussi de celle d'une association professionnelle. Or, qu'en est-il de ces visions? Dans quelle mesure coïncident-elles pleinement? Ce texte propose d'aborder ces 
questions à partir de la formation en grand paysage. Après avoir présenté l'atelier pédagogique en grand paysage du premier cycle puis les facteurs qui ont contribué à la mise en place d'un programme de second cycle, il met en relation ce dernier avec les exigences posées par l'AAPC pour l'accréditation. Cette mise en relation soulève des enjeux qui, au-delà du grand paysage, sont au cœur de la formation et de la profession.

In Quebec, teaching landscape architecture presents certain singularities. The only training available is provided by programmes offered at Montreal University and the professional graduate programme is subject to accreditation by the Canadian Association of Landscape Architects (in French, Association des architectes paysagistes du Canada, AAPC). At university such a situation is singular since the programme derives not only from the perspective of the teachers but also from that of a professional association. What are these perspectives? To what extent do they coincide? This article seeks to address these questions from the angle of training in the larger landscape. After presenting the training workshop of the larger landscape in the undergraduate programme, it describes the factors which led to the creation of the graduate programme, and examines the latter in the light of the requirements for AAPC accreditation. This analysis raises questions which, beyond the scope of the larger landscape, lie at the heart of the landscape architecture profession and the way landscape architecture is taught.

\section{INDEX}

Mots-clés : grand paysage, architecture de paysage, atelier pédagogique, formation professionnelle, accréditation

Keywords : larger landscape, landscape architecture, pedagogical workshop, vocational training, accreditation

\section{AUTEUR}

\section{GÉRALD DOMON}

Gérald Domon est professeur titulaire et directeur scientifique associé à la chaire en paysage et environnement de l'université de Montréal.

gerald.domon[at]umontreal[dot]ca 